\title{
Arrays of elliptical Fe(001) nanoparticles: Magnetization reversal, dipolar interactions, and effects of finite array sizes
}

\author{
Maj Hanson* \\ Department of Applied Physics, Chalmers University of Technology, SE-412 96 Göteborg, Sweden \\ Rimantas Bručas \\ Department of Engineering Sciences and Ångström Microstructure Laboratory, Uppsala University, \\ Box 534, SE-751 21 Uppsala, Sweden \\ Tomasz J. Antosiewicz \\ Centre of New Technologies, University of Warsaw, Banacha 2c, 02-097 Warszawa, Poland \\ and Department of Applied Physics, Chalmers University of Technology, SE-412 96 Göteborg, Sweden \\ Randy K. Dumas \\ Physics Department, University of Gothenburg, SE-412 96 Göteborg, Sweden \\ Björgvin Hjörvarsson \\ Department of Physics and Astronomy, Uppsala University, Box 516, SE-751 20 Uppsala, Sweden \\ Vegard Flovik and Erik Wahlström \\ Department of Physics, Norwegian University of Science and Technology, N-7491 Trondheim, Norway
}

(Received 19 May 2015; published 22 September 2015)

\begin{abstract}
The magnetic properties of arrays of nanoparticles are determined by the interplay between the individual particle properties and the dipolar interactions between them. Here we present a study of arrays of elliptical $\mathrm{Fe}(001)$ particles of thickness $10-50 \mathrm{~nm}$. The aspect ratios of the ellipses are 1:3, their short axes $a=50,100$, or $150 \mathrm{~nm}$, and the periodicity of the rectangular arrays is either two or four times the corresponding axes of the ellipses. Magnetic measurements together with numerical and micromagnetic calculations yield a consistent picture of the arrays, comprising single-domain nanoparticles. We show that the magnetization reversal, occurring in the range 100-400 $\mathrm{mT}$ for fields applied along the long axis, is mainly determined by the properties of the corresponding single Fe ellipses. The interaction fields of the order of tens of $\mathrm{mT}$ can be tuned by the array configurations. For the actual arrays the interactions promote switching. For film thicknesses below the Bloch wall width parameter of $\mathrm{Fe}, l_{\mathrm{w}}=22 \mathrm{~nm}$, magnetization reversal occurs without formation of domain walls or vortices. Within this range arrays may be tuned to obtain a well-defined switching field. Two general conclusions are drawn from the calculations: the character of the interaction, whether it promotes or delays magnetization reversal, is determined by the aspect ratio of the array grid, and the interaction strength saturates as the size of the array increases.
\end{abstract}

DOI: 10.1103/PhysRevB.92.094436

\section{INTRODUCTION}

The physics of arrays of small ferromagnetic particles is a topic of great interest in current research. Part of the motivation for studies of such systems lies in their potential for applications, e.g., for data storage [1], as discussed within the developing field of magnonics [2,3], or for studies of artificial spin ices [4]. The arrays are complex systems in which the individual particles as well as the interparticle interactions, introduced by the design of the arrays, must be controlled to obtain any desired static and dynamic magnetic properties of a device.

\footnotetext{
*maj.hanson@chalmers.se

Published by the American Physical Society under the terms of the Creative Commons Attribution 3.0 License. Further distribution of this work must maintain attribution to the author(s) and the published article's title, journal citation, and DOI.
}

PACS number(s): 75.60.-d, 75.75.-c

In a finite two-dimensional (2D) array of magnetic particles the dipolar interactions depend on the symmetry and size of the array. Finite arrays of single domain, weakly interacting magnetic dots were investigated by Stamps and Camley [5]. They found that a resulting array anisotropy, depending on array size as well as symmetry, was induced and that this in turn controlled the hysteresis curves (comprising discrete steps) and magnetization reversal processes. Kayali and Saslow [6] applied the same model in studies extended to larger arrays and Takagaki and Ploog [7] performed similar studies adding an internal magnetocrystalline anisotropy to the individual particles. In a comment Alcántara Ortigoza et al. [8] pointed out that some qualitative differences between the results obtained in the two last mentioned studies could be attributed to different choices of the dipolar interaction strength in respective work. The ground state of two-dimensional lattices attracts continued interest and, as discussed in a recent work by Ewerlin et al. [9], its nature depends on details of the interaction and boundary conditions. 
Realistic array models require the intrinsic properties and magnetic switching of the individual particles to be included in the calculations. As for the choice of materials, permalloy $\left(\mathrm{Fe}_{81} \mathrm{Ni}_{19}\right)$ is the archetype for soft magnetic properties with low intrinsic anisotropy. Single-domain permalloy particles of circular or other shapes with low aspect ratio are often used as model systems to study the influence of array configurations. As methods for fabrication of nanostructures [10] and computation capacity develop, the number of publications in the field continues to increase. It is beyond the scope of this paper to give a comprehensive review of the current state; here we give a few examples in which also further references can be found. A system that attracted particular interest comprises nearly isotropic permalloy particles, which for a certain range of thicknesses and lateral sizes may undergo switching through vortex formation and movement. The properties of the particles were reviewed by Guslienko [11], and their sensitivity to dipolar interactions was studied for different array configurations, e.g., by Novosad et al. [12]. Also arrays with elliptical permalloy particles were studied, but to a lesser extent; see, e.g., the work by Wang et al. [13], Pardavi-Horvath [14], and references therein.

For arrays of materials with higher intrinsic magnetization and anisotropy the classic ferromagnet $\mathrm{Fe}$ is well suited, and such systems are less well studied. In earlier work we developed fabrication methods and studied the thickness and size dependence of the equilibrium domain structures in rectangular and circular particles of epitaxial $\mathrm{Fe}(001)$ films [15-17]. With the aim to study the interplay between singleparticle properties and array configurations, we prepared two-dimensional (2D) arrays of smaller, single-domain (SD) elliptical particles in the same way. Magnetization measurements together with numerical and micromagnetic calculations in the quasistatic regime show how the field $B_{\text {sw }}$ for magnetization reversal depends on film thickness and lateral extension of the individual particles, and how the interaction strength $B_{\mathrm{i}}$ and its character varies with the design of the arrays. Balancing $B_{\mathrm{sw}}$ and $B_{\mathrm{i}}$ makes it possible to tune the onset of switching of the arrays.

The paper is organized as follows. Section II describes the sample preparation and methods for magnetic characterization. Section III presents magnetic hysteresis, dc remanent magnetization observed by magnetic force microscopy (MFM), first-order reversal curves (FORCs), micromagnetic simulation of single particle switching, numerical analysis of the interaction field in finite array configurations, and micromagnetic calculations of magnetization reversal in finite arrays. Section IV gives account of the arguments behind our interpretation regarding magnetization reversal and influence of array configurations. Section $\mathrm{V}$ gives a summary of the main results of the work.

\section{MATERIALS AND METHODS}

\section{A. Sample preparation}

Fe films with thickness $t$ in the range $10 \mathrm{~nm} \leqslant t \leqslant 50 \mathrm{~nm}$ were epitaxially grown by magnetron sputtering on $\mathrm{MgO}(001)$ substrates, $10 \times 10 \times 0.5(\mathrm{~mm})^{3}$. The films were capped by a 5-nm layer of $\mathrm{Al}_{2} \mathrm{O}_{3}$ to prevent oxidation. Samples with arrays of elliptical particles were patterned by electron-beam

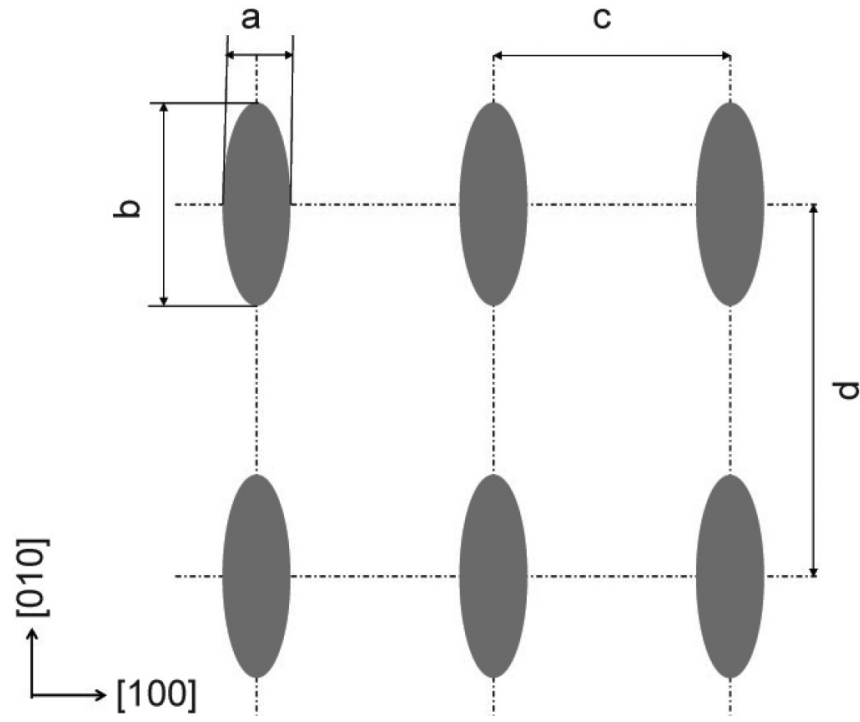

FIG. 1. Layout for patterning of arrays of ellipses. Ellipses with $a=50,100$, or $150 \mathrm{~nm}$ were prepared with the axes along the in-plane easy magnetization directions [100] and [010] of the $\mathrm{Fe}(001)$ film. $b=3 a$. The center-to-center distances between the particles scale with the sizes of the ellipses. For narrow and wide distances $c=2 a$ and $d=2 b$ or $c=4 a$, and $d=4 b$, respectively.

lithography and ion-beam milling, as described earlier [15]. The axes of the ellipses are oriented along the easy directions of magnetization [100] and [010] in Fe; see the sketch of the layout in Fig. 1. The aspect ratio $a: b$ is $1: 3$ for all ellipses and the center-to-center interparticle distances are either two (narrow separation) or four times (wide separation) the lateral size of the corresponding ellipses. On each film up to six samples, including one circular reference with diameter $1.7 \mathrm{~mm}$, were prepared. A patterned area is composed of a number of $e$-beam fields with area $500 \mu \mathrm{m} \times 500 \mu \mathrm{m}$, that form samples of rectangular areas with edges up to $3 \mathrm{~mm}$. A sample comprises of the order of $5 \times 10^{6}-10^{8}$ ellipses.

\section{B. Magnetic measurements}

The magnetization of the samples was measured with an alternating gradient magnetometer (AGM) from Princeton Measurements Corporation. The samples were investigated at room temperature in magnetic fields $B$ in the range $-2 \mathrm{~T} \leqslant B \leqslant 2 \mathrm{~T}$. Hysteresis curves and FORCs were recorded. The magnetization $M$ was obtained after diamagnetic correction determined from the slope of the high-field hysteresis curves. It was verified that the saturation magnetization $M_{s}$ of the arrays and reference samples were equal to the value for the nominal amount of $\mathrm{Fe}$.

The arrays were studied by scanning force microscopy (SFM) in a Dimension3000 from Digital Instruments. Topographic and magnetic images were recorded at room temperature in zero field. The magnetic tip used for imaging has a coating of $\mathrm{Co}$ and $\mathrm{Cr}$ films yielding a resulting radius of curvature of the order of $90 \mathrm{~nm}$. The switching behavior was studied during dc demagnetization, by scanning sampled areas and counting the number of switched particles in arrays that were first saturated in a field $B=+2 \mathrm{~T}$, applied along the long 
axis of the ellipses, and subsequently exposed to a field in the opposite direction. Due to tip-sample interactions and limited resolution of dense-packed patterns the demagnetization was not traced for all samples.

\section{Micromagnetic simulation}

The field dependence of the magnetic state of the $\mathrm{Fe}$ ellipses was calculated using the MicroMagus package for quasistatic micromagnetic simulations [18]. Each ellipse was discretized into layers of cubic elements. In order to obtain mesh independence the discretization cells should have sides of the same order, or better, less than, the two characteristic length scales in micromagnetics (both frequently called exchange lengths). The competition between exchange and dipolar energy is characterized by the magnetostatic exchange length $l_{\mathrm{ex}}=\left(\frac{A}{K_{\mathrm{d}}}\right)^{1 / 2}$ and the competition between exchange and anisotropy energies is characterized by the Bloch wall width parameter $l_{\mathrm{w}}=\left(\frac{A}{K_{1}}\right)^{1 / 2}$ [19-22]. Here $A$ is the exchange stiffness constant, $K_{\mathrm{d}}$ is the energy density of the stray field, and $K_{1}$ is the first-order anisotropy constant. An upper limit for $K_{\mathrm{d}}$ is $\frac{1}{2} \mu_{0} M_{\mathrm{s}}^{2}$, where $\mu_{0}$ is the permeability of vacuum and $M_{S}$ is the saturation magnetization. In the simulation we used the following bulk values for Fe: $A=21 \times 10^{-12} \mathrm{~J} \mathrm{~m}^{-1}$, $M_{\mathrm{s}}=1.7 \times 10^{6} \mathrm{~A} \mathrm{~m}^{-1}, K_{1}=4.3 \times 10^{4} \mathrm{~J} \mathrm{~m}^{-3}$. These yield $l_{\mathrm{w}}=22 \mathrm{~nm}$ and $l_{\mathrm{ex}}=3.5 \mathrm{~nm}$. An estimate of the width of a Bloch wall in Fe, $\delta_{\mathrm{w}}=64 \mathrm{~nm}$, is given by Coey [22]. For the smallest ellipses simulations made with cube sides of 2 and $3 \mathrm{~nm}$, respectively, yielded the same switching behavior. For the rest of the simulations we used cubic cells with sides $\Delta_{x}=\Delta_{y}=\Delta_{z}=3 \mathrm{~nm}$. Here the $x$ and $y$ axes are along the short and long axes of the ellipses, respectively, and the $z$ direction is perpendicular to the film plane. To minimize computation time the number of discretization cells should be factors of low prime numbers. For example, ellipses $50 \mathrm{~nm} \times 150 \mathrm{~nm}$ with $t=10 \mathrm{~nm}$ are simulated with $a=16 \Delta_{x}$, $b=48 \Delta_{y}$, and $t=3 \Delta_{z}$.

\section{RESULTS}

\section{A. Thickness and size dependence of magnetization reversal in arrays}

To study the thickness and size dependence of the arrays, the samples were first characterized by measuring the hysteresis curves, and by MFM observation of the ellipses after dc demagnetization. Figure 2 gives an overview of hysteresis curves of arrays within the actual size and thickness range. Three different sizes of ellipses and three thicknesses are displayed. The center-to-center separation is narrow (twice the corresponding lateral size of the ellipses). For all three sizes the 30-nm particles are hardest to switch. The hysteresis curves of the two largest and thickest particles, with short axes 100 and $150 \mathrm{~nm}$ and $t=50 \mathrm{~nm}$, are characteristic for magnetization reversal through domain-wall formation and movement. The curves for samples with $t=10$ and $30 \mathrm{~nm}$ have different shapes, and we will show that in these magnetization reversal occurs through formation and movement of quasisingle domain configurations called $S$ and $C$ states [23]. The reversal processes will be further analyzed by micromagnetic simulations and FORC analysis.

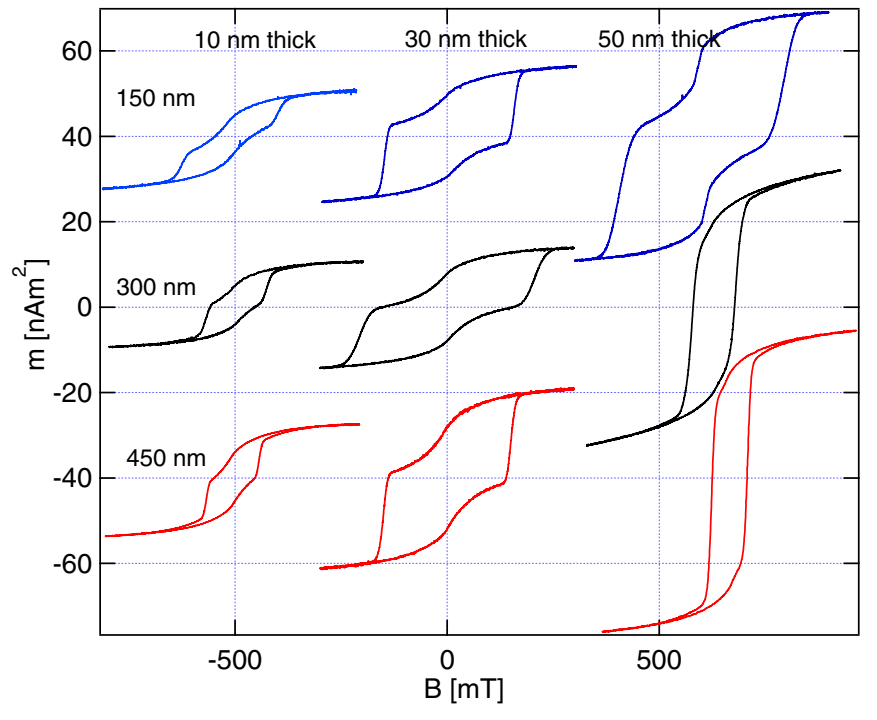

FIG. 2. (Color online) Size and thickness dependence of hysteresis curves of Fe ellipses with the field applied along the long axes. The interparticle separation is twice the lateral size of corresponding ellipses. The film thicknesses and the long axes are noted in the figure. All curves are drawn to scale with an ofset for all except the central one.

In a second set of arrays, ellipses of size $50 \mathrm{~nm} \times 150 \mathrm{~nm}$ and $100 \mathrm{~nm} \times 300 \mathrm{~nm}$ were prepared with $t=10,20,30$, and $50 \mathrm{~nm}$. To study the influence of interactions, samples with narrow and wide (four times the size of the ellipses) particle separation were made. All samples are hardest to switch for $t=20 \mathrm{~nm}$. The thickness dependence of the hysteresis is shown for $100 \mathrm{~nm} \times 300 \mathrm{~nm}$ ellipses with $400 \mathrm{~nm} \times$ $1200 \mathrm{~nm}$ separation in Fig. 3(a). The field region with the steep slope right until the loop closes is the range where the dominant magnetization component, along the long axis of the ellipses, is reversed. This is clearly seen in a comparison with the stepwise dc demagnetization of the sample with $t=20 \mathrm{~nm}$; see Fig. 4. The MFM images of the remanent state after saturation display particles of elliptical shape with stray fields of dipolar character, aligned along the saturation field. This is characteristic for a single domain (SD) state, which is without domain walls, but not necessarily with homogeneous magnetization. In the ellipses edge domains are formed to minimize the stray fields. All investigated samples follow a similar switching behavior as observed in Fig. 4. During the stepwise dc demagnetization the particles were always observed in one or the other dipolar state and the switching began with isolated particles at random positions. In the ac demagnetized state, however, the largest particles were occasionally observed in another state, e.g., the particles of size $100 \mathrm{~nm} \times 300 \mathrm{~nm}$ were observed in a bidomain state for $t=30 \mathrm{~nm}$ and thicker, but not for $t=10 \mathrm{~nm}$. The smallest $50 \mathrm{~nm} \times 150 \mathrm{~nm}$ ellipses were always observed as single domains. Thus, from the magnetic force microscopy we conclude that the particles form stable single domains and there is no long-range ordering in the arrays.

The shape of the hysteresis curves have widely differing characters; pot bellies with spreading middles, and wasp waists with constrained middles [24] can be seen, as well as curves 


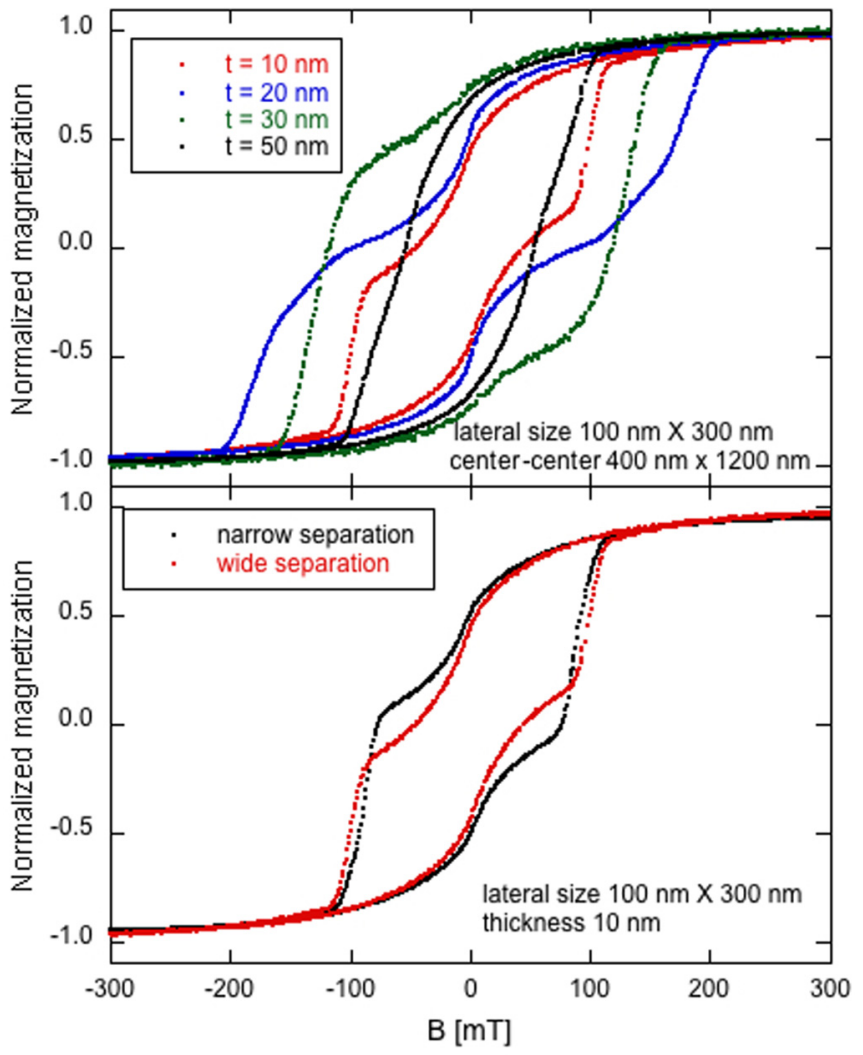

FIG. 3. (Color online) (a) The thickness dependence of hysteresis curves for Fe elliptical particles with lateral size and thicknesses as noted in the figure. Note the order of complete reversal: $t=50$, 10,30 , and $20 \mathrm{~nm}$. (b) The influence of interactions for the 10-nm sample in (a). The field is applied along the long axis.

with a character like any polycrystalline ferromagnetic film. For these the usual coercivity $B_{\mathrm{c}}$ defined as the field at $M=$ 0 , is not a proper measure of the switching field, thus, for comparison the closing of the hysteresis curve at $\mathrm{B}_{\mathrm{cl}}$ is used. Samples with narrow and wide separation yield very similar hysteresis curves; see Fig. 3(b). As a main influence of stronger interactions, it was found that the switching starts earlier and is completed at about the same value of $\mathrm{B}_{\mathrm{cl}}$ as the sample with weaker interaction. For the samples with wide separation of $100 \mathrm{~nm} \times 300 \mathrm{~nm}$ ellipses the values of $\mathrm{B}_{\mathrm{cl}}$ are in the range 100-200 mT. For the $50 \mathrm{~nm} \times 150 \mathrm{~nm}$ ellipses (not shown here) the corresponding range is $200-400 \mathrm{mT}$. The influence of interactions is further analyzed from the FORC curves, and numerical calculations.

In a third set of arrays ellipses of size $100 \mathrm{~nm} \times 300 \mathrm{~nm}$ and $150 \mathrm{~nm} \times 450 \mathrm{~nm}, t=15,25$, and $30 \mathrm{~nm}$, and wide separation were prepared. Of these the $25-\mathrm{nm}$ sample has the highest values of $B_{\mathrm{cl}}$. The hysteresis curves for the $150 \mathrm{~nm} \times 450 \mathrm{~nm}$ ellipses are shown in Fig. 5 and the corresponding dc remanent switching range in shown in Fig. 6. Both measurements indicate the same values of $B_{\mathrm{cl}}$, being in the range 100-200 mT. When comparing the switching field $B_{\text {sw }}$ determined by micromagnetic simulation for an individual Fe ellipse (cf. Fig. 7), the experimentally determined value $B_{\mathrm{cl}}$ for an array is lower. The differences, as well as range of switching, vary between different samples. For the $150 \mathrm{~nm} \times$

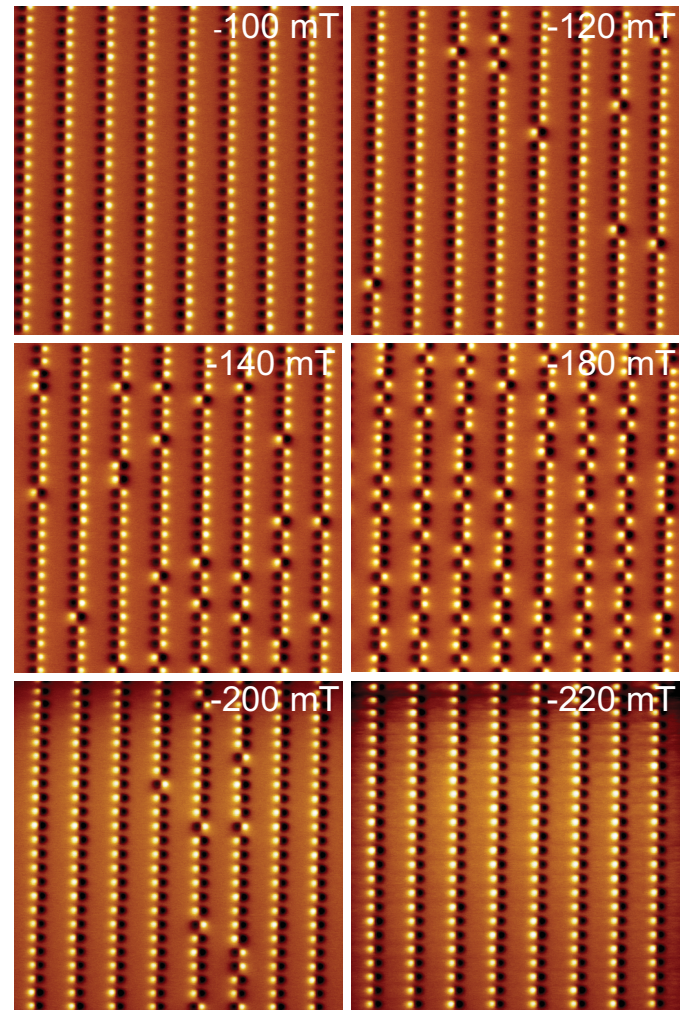

FIG. 4. (Color online) MFM images taken during stepwise dc demagnetization of the saturated state of an array of 20-nm-thick ellipses with lateral size $100 \mathrm{~nm} \times 300 \mathrm{~nm}$ and the separation four times the lateral size of the ellipses. The magnetization was made $e x$ $s i t u$, thus the images do not represent identical areas. The sampling is made at random locations in the central part of the sample. The scan sizes are $10 \mu \mathrm{m} \times 10 \mu \mathrm{m}$.

$450 \mathrm{~nm}$ ellipses, see Figs. 5 and 6, the values of $B_{\mathrm{cl}}$ are about $60 \mathrm{mT}$ lower than the calculated $B_{\mathrm{sw}}$ for corresponding single Fe ellipses with thicknesses 15, 25, and $30 \mathrm{~nm}$. For the $100 \mathrm{~nm} \times 300 \mathrm{~nm}$ ellipses made of the same films (not shown here), the corresponding difference is about $50 \mathrm{mT}$.

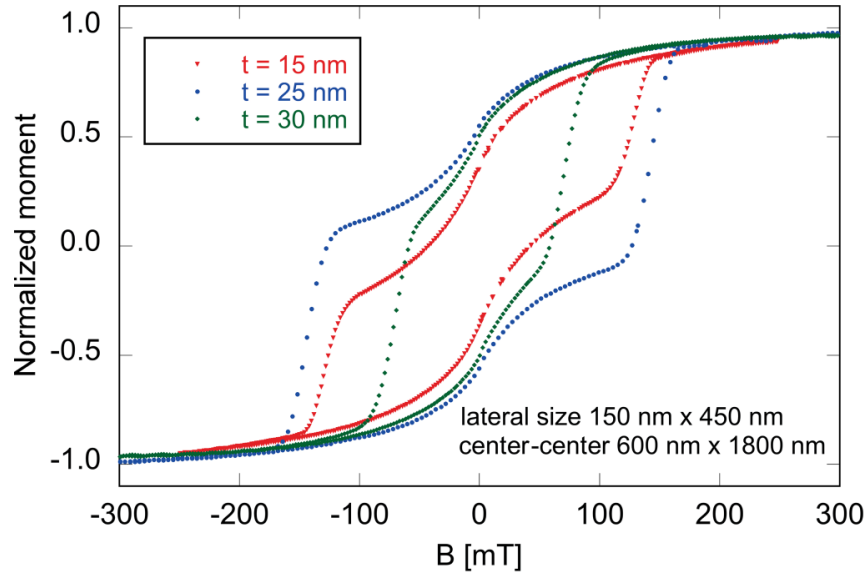

FIG. 5. (Color online) The thickness dependence of the hysteresis curves for Fe elliptical particles. The lateral size and film thicknesses are noted in the figure. The field is applied along the long axis. Note the order of complete reversal: $t=30,15$, and $25 \mathrm{~nm}$. 


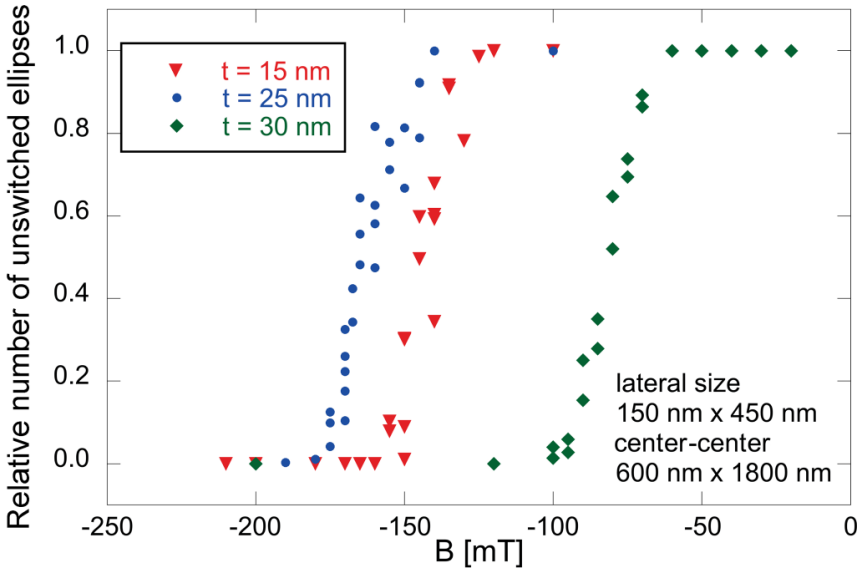

FIG. 6. (Color online) The relative number of ellipses that remained unswitched vs a reversal field applied after saturation in a field of $+2 \mathrm{~T}$. The lateral size and film thicknesses are noted in the figure. Note the order of complete reversal: $t=30,15$, and $25 \mathrm{~nm}$.

A common trait of the hysteresis curves is an initial curved slope as the field decreases to a remanent magnetization $M_{r}$ significantly lower than $M_{s}$. Thus they deviate from the ideal square hysteresis behavior. This is mainly due to the initial film properties and demagnetizing effects in the particles, and does not significantly affect the switching of the ellipses, which occurs in a different field range. To estimate orders of magnitude, we take the demagnetizing factors from the relations applied in our earlier work [15] and obtain for the largest ellipses the values along the long axes; $N_{y}=0.025$, 0.041 , and 0.050 , for $t=15,25$, and $30 \mathrm{~nm}$ respectively. The ratios $M_{r} / M_{s}=0.46,0.59$, and 0.47 taken from the hysteresis curves in Fig. 5 increase to 0.64, 0.70, and 0.68, respectively, after demagnetization correction.

The switching field of the arrays display a systematic dependence of the thickness and size of the individual elements. This will be compared with the switching properties of the corresponding single Fe ellipses, which are analyzed in the next section.

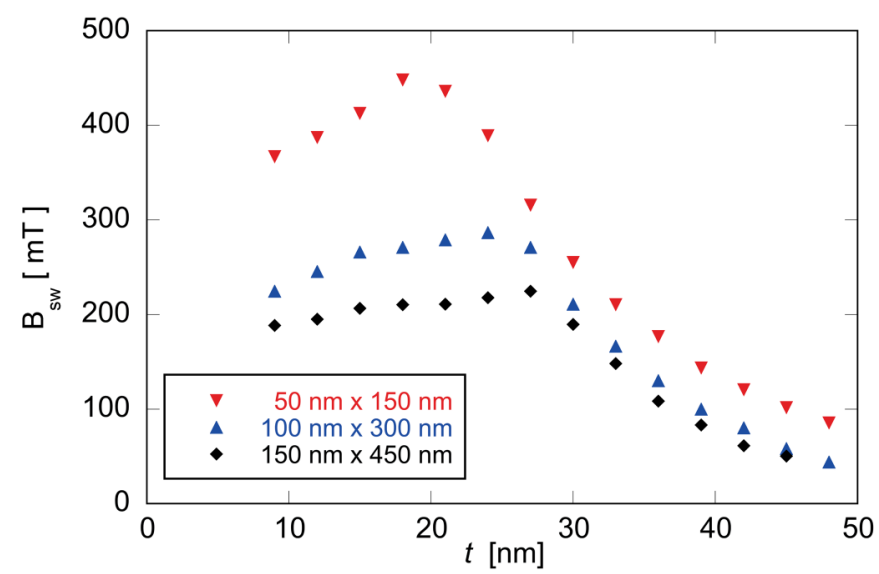

FIG. 7. (Color online) Micromagnetic simulation of the field $B_{\mathrm{sw}}$ for magnetization reversal of a single Fe ellipse, plotted vs film thickness $t$. The field is applied along the long axis of the ellipses. The lateral sizes of the ellipses are noted in the figure.

\section{B. Micromagnetic simulation of magnetization reversal in single Fe ellipses}

The field dependence of the magnetization of the Fe ellipses was studied in micromagnetic simulations. By treating the ellipses as structures composed of 3-nm-thick layers it is possible to follow the behavior of the individual layers. The switching field $B_{\mathrm{sw}}$ for a single Fe ellipse was simulated by starting with the single ellipse saturated in a field $+B_{i}$ applied along the easy axis of the ellipse, and then recording the magnetic equilibrium state as the field was decreased in steps until the magnetization was completely reversed. It is not possible to catch the reversal in a quasistatic simulation; the value of $B_{\mathrm{sw}}$ is taken as the first field step in showing a switched state. Figure 7 shows the thickness dependence of the switching for three lateral sizes of ellipses. $B_{\mathrm{sw}}$ has a maximum at $t_{\mathrm{sw}}$, in the range of the wall width parameter $l_{\mathrm{w}}=$ $22 \mathrm{~nm}$. This marks a transition between different magnetization reversal processes. For thicknesses below $t_{\mathrm{sw}}$ all layers undergo identical in-plane reversals, and the micromagnetic state of a single ellipse immediately before the final switching of the dominant magnetization direction is an $S$-like quasisingle domain state. Figure 8 shows the in-plane components $m_{x}$ and $m_{y}$ and the out-of-plane component $m_{z}$ in the field steps before and after switching of layer 1 of three. $m_{x}$ and $m_{y}$ are identical in all three layers. The $m_{z}$ components are practically zero, except at the edges of the long axis of layers 1 and 3 , which have equally large components of reversed signs, that is opposite directions. As the thickness increases, a phase shift between the in-plane rotations in different layers can be observed, together with an increasing out-of-plane component. Only in the thickest films and for the two largest particle sizes were states with clear domain walls observed during switching;

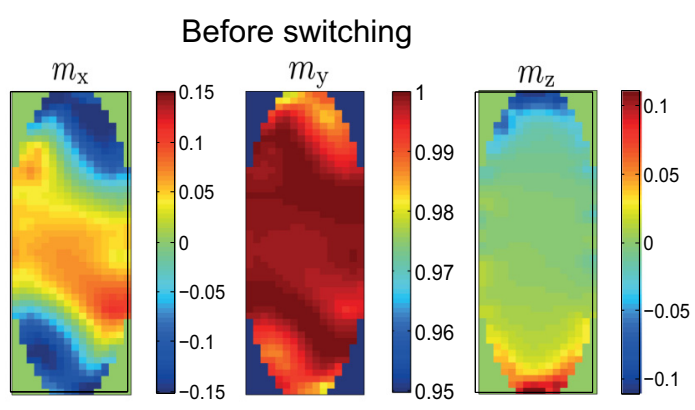

After switching

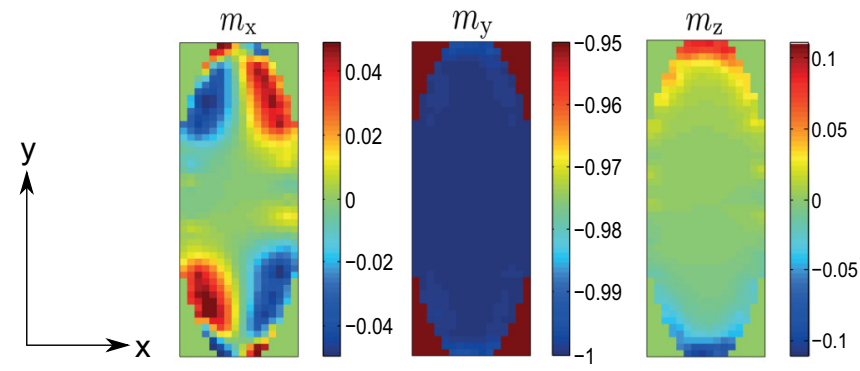

FIG. 8. (Color online) Micromagnetic simulation of a single $\mathrm{Fe}(001)$ ellipse with easy axes along the [100] and [010] directions. The field is applied along the long axis. In the simulation the thickness was $3 \times 3 \mathrm{~nm}$, the short axis $16 \times 3 \mathrm{~nm}$, and the long axis $48 \times 3 \mathrm{~nm}$. Layer 1 of three is plotted. 
for $100 \mathrm{~nm} \times 300 \mathrm{~nm}$ with $t=48 \mathrm{~nm}$ and for $150 \mathrm{~nm} \times 450 \mathrm{~nm}$ with $t \geqslant 45 \mathrm{~nm}$.

The simulated thickness dependence of the switching field is in good agreement with the experimental observations, which showed a maximum for $t=20-25 \mathrm{~nm}$. In general the hysteresis and dc remanent demagnetization are softer than the calculated values indicate. There are several factors that can lead to such discrepancies, among which effects of disturbed particle edges and interactions are obvious. The program allows simulations of edge defects by setting an area near the element border where the magnetization is reduced. The values of $B_{\mathrm{sw}}$ displayed in Fig. 7 were obtained with a width of the disturbed area corresponding to one calculation cell. With a disturbance of for example two cells width, the value of $B_{\text {sw }}$ is decreased by about $13 \mathrm{mT}$ for the smallest and thinnest ellipses.

Two important results from the magnetic measurements are (i) the thickness dependence of the switching field for a given pattern shows the characteristics of the corresponding single Fe particles with a clear maximum in the range around $t=20-25 \mathrm{~nm}$, and (ii) the switching starts in lower fields when the interactions are increased.

The following sections treat the interactions in the arrays.

\section{Interactions in first-order reversal curves FORCs}

\section{Experimental details}

In order to investigate the details of the magnetization reversal processes and in particular to gain an experimental measure of the strength of the magnetostatic interactions present within the array, the first-order reversal curve (FORC) technique [25-27] was used. With the field $H$ applied along the long axes of the ellipses the measurement of a FORC proceeds as follows: After positive saturation, the applied field is reduced to a given reversal field, $H_{R}$. From this reversal field the magnetization is then measured back towards positive saturation. This process is repeated for decreasing reversal fields filling the interior of the major hysteresis loop with a family of FORCs, Figs. 9(a), 9(c), and 9(e), where the magnetization is a function of both the applied and reversal fields, $M\left(H, H_{R}\right)$. The FORC distribution is then defined [25] as a mixed second-order derivative of the normalized magnetization:

$$
\rho\left(H, H_{R}\right) \equiv-\frac{1}{2} \frac{\partial^{2} M\left(H, H_{R}\right) / M_{S}}{\partial H \partial H_{R}} .
$$

It is often convenient to perform a simple coordinate transformation and interpret the results in terms of a local coercivity $H_{C}=\left(H-H_{R}\right) / 2$ and bias/interaction field $H_{B}=$ $\left(H+H_{R}\right) / 2$, as shown in Figs. 9(b), 9(d), and 9(f).

\section{Analysis}

The family of FORCs and corresponding FORC distribution for the median sized $(100 \mathrm{~nm} \times 300 \mathrm{~nm}), 30$-nm-thick Fe ellipses on a $400 \mathrm{~nm} \times 1200 \mathrm{~nm}$ grid are shown in Figs. 9(a) and 9 (b), respectively. While the major loop, as seen by the outer boundary of the family of FORCs, shows a distinct pinching often seen in magnetic nanostructures that reverse via a vortex state [26], the FORC distribution shows no signs of vortex state reversal. In fact, the FORC distribution, which is characterized by a relatively narrow ridge centered along
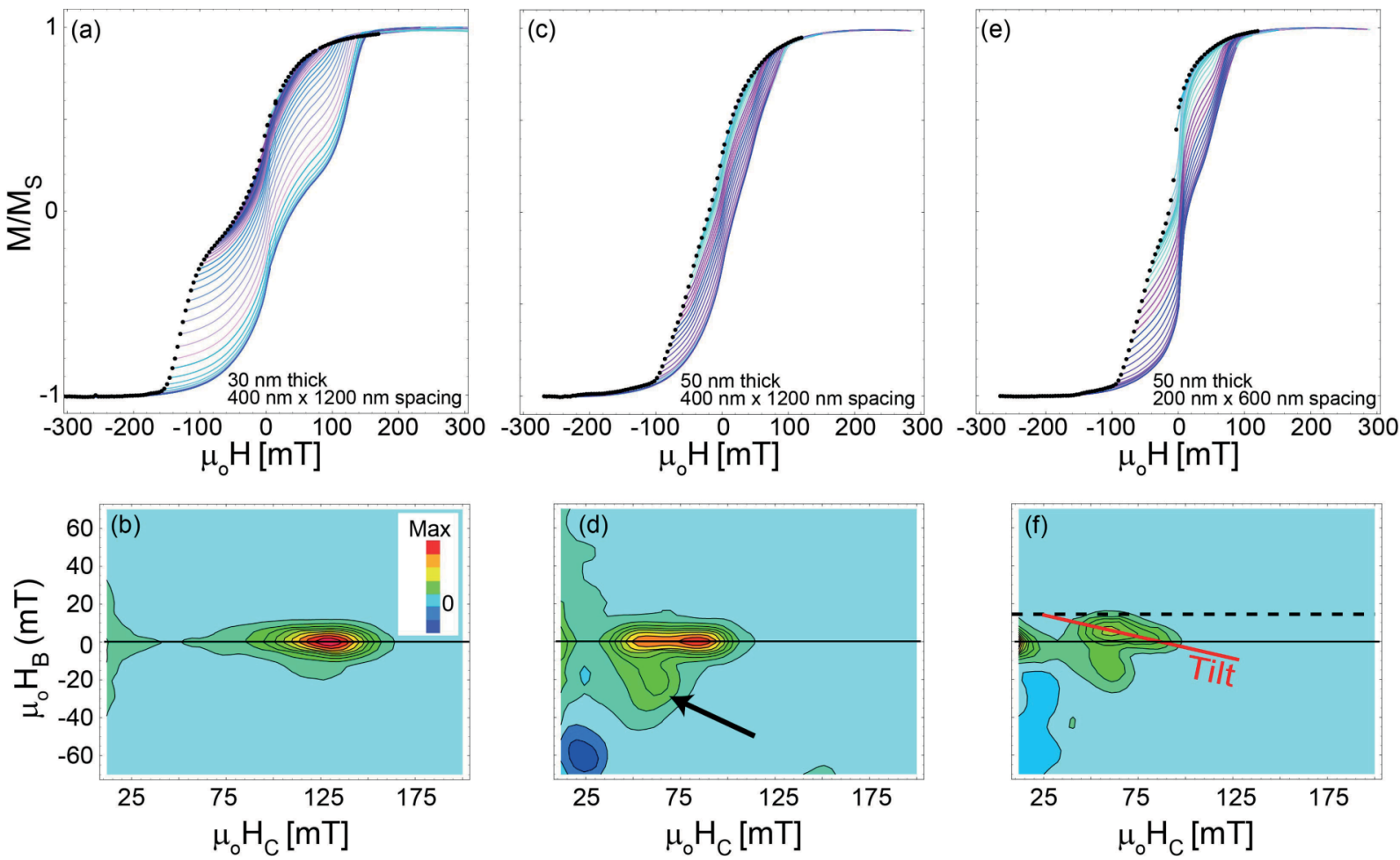

FIG. 9. (Color online) Families of FORCs, whose starting points are represented with black dots (a, c, e) and corresponding FORC distributions (b, d, f) plotted against $\left(H_{C}, H_{B}\right)$ coordinates for $100 \mathrm{~nm} \times 300 \mathrm{~nm}$ Fe ellipses with the indicated thickness and grid spacing. 
the $H_{C}$ axis, is consistent with a single highly irreversible switching of the magnetization in each ellipse. However, the relatively low remanence of the major loop is consistent with a significant amount of curling or buckling preceding the switching. The extent of the ridge along the $H_{C}$ axis provides a measure of the coercivity distribution while the fact that this ridge is not skewed in any manner along the $H_{B}$ axis indicates that the ellipses are highly noninteracting. The FORC distribution begins to evolve as the thickness of the Fe ellipses is increased to $50 \mathrm{~nm}$, Figs. 9(c) and 9(d), for the same grid spacing. The average interaction field of the ellipses should increase as they get thicker, which is observed as the FORC distribution begins to develop a clear feature for negative values along the $H_{B}$ axis, indicated with an arrow. However, the primary ridge still lies predominately along the $H_{C}$ axis $\left(H_{B}=0\right)$ indicating that the interactions are still quite weak. The interactions can be increased further by decreasing the spacing of the ellipses to $200 \mathrm{~nm} \times 600 \mathrm{~nm}$, as shown in Figs. 9(e) and 9(f). Here the FORC distribution, Fig. 9(f), not only has developed a significant feature for negative values of $H_{B}$, but the ridge along the $H_{C}$ axis now has a significant tilt with respect to the $H_{C}$ axis. Following the analysis in earlier work [27], the maximal extent of this tilt along the positive $H_{B}$ axis, shown with a horizontal dashed line, provides a direct experimental measure of the average interaction fields, namely $\mu_{0} H_{i} \approx 18 \pm 2 \mathrm{mT}$. This is in good agreement with the value of the interaction field $22.6 \mathrm{mT}$ that was calculated at the center of a $61 \times 61$ array of the same ellipses. The calculation was made as described in the following section.

\section{Calculation of interaction fields in arrays}

The interaction between the nanoparticles is investigated by calculating the magnetic dipolar fields at different positions in the arrays. We consider the saturated case where each nanomagnet is assumed to have a magnetic moment equal to the magnetization of iron $M_{\mathrm{Fe}}=1.7 \times 10^{6} \mathrm{~A} / \mathrm{m}$ times the volume $V$. To calculate the interaction fields with high spatial accuracy we divide each ellipse into tiny cubes with $\Delta_{x}=\Delta_{y}=\Delta_{z}=2 \mathrm{~nm}$. The considered magnetization is a static quantity. Each cube behaves like a tiny magnetic dipole with moment $\mathbf{m}$ and and generates a magnetic near-field according to

$$
\mathbf{B}=\frac{\mu_{0}}{4 \pi r^{3}}[3 \mathbf{n}(\mathbf{n} \cdot \mathbf{m})-\mathbf{m}],
$$

where $\mathbf{n}$ is a unit vector pointing from the cube to the place at which we calculate the field and $\mu_{0}$ is the permeability of free space.

The ellipses as well as the periodicity have an aspect ratio of 3 and here we calculate the field from a square pattern of $61 \times 21$ particles occupying $6 \mu \mathrm{m} \times 6 \mu \mathrm{m}$ with $100 \mathrm{~nm}$ being the short axis periodicity. We calculate the magnetic field in the center of the array, at the corners and either edge. The positions are marked in Fig. 10. Equation (2) is used to calculate the magnetic field at desired positions for discretized ellipses from 10 to $50 \mathrm{~nm}$ in thickness and semiaxes 25 and $75 \mathrm{~nm}$.

We begin by analyzing the magnetic field that is generated by the saturated nanomagnets. In the calculations we assume

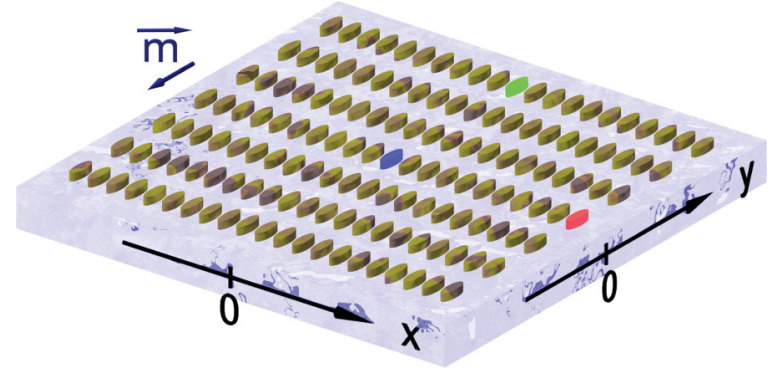

FIG. 10. (Color online) Array scheme for the calculations. A nanomagnet is in the shape of an ellipse of a given height and semiaxes 25 and $75 \mathrm{~nm}$ in the $x$ and $y$ directions, respectively. The periodicity has the same aspect ratio as that of the semiaxes and equals 100 and $300 \mathrm{~nm}$. The magnetic moment of each ellipse is assumed to be in the $-y$ direction. We mark clearly the particles for which we calculate the magnetic fields acting onto them from the remainder of the array, i.e., we calculate the magnetic field at its geometrical center as if it were not present; these particles are the central particle, those at the top edge (along the $x$ axis), and the side edge ( $y$ axis).

that the magnetic dipole moment is in the $-y$ direction. The interaction magnetic field is calculated at the geometrical center of each ellipse in question, i.e., the field acting onto the central ellipse is calculated as a sum of the fields from all ellipses except for the central one. We begin by investigating the spatial dependence of the field for 10-nm-thick ellipses in an array with a short axis periodicity of $100 \mathrm{~nm}$. The results are plotted in Fig. 11. The field acting onto the central ellipse, and indeed onto most of the ellipses in the array, is approximately $10 \mathrm{mT}$. However, at the edges it deviates from this value and at each of the four corners, in Fig. 10, it is equal to $6 \mathrm{mT}$. At the side edge the field decays down to $4 \mathrm{mT}$ (in the geometrical center of that edge), which is the lowest calculated value. The opposite happens at the top edge which has a dense arrangement of ellipses-the field there reaches a value of $13.5 \mathrm{mT}$. These are the $B_{y}$ components which are aligned along the magnetization vector and add/subtract from the outside magnetic field applied to switch the magnetization of the ellipses. In addition to the dominant $B_{y}$ component, at the center of the ellipses one can determine the cross-polarized $B_{x}$ component. It is much weaker reaching a value of $-2 \mathrm{mT}$ at the corner (the sign depends on which corner) and for most of the array is negligible in the geometrical centers of the ellipses due to symmetry. As noted, these interaction fields are calculated at the geometrical centers of the ellipses. However, these fields within the volume of an ellipse will inevitably vary. In Fig. 12 we present the dependence of the magnetic field generated by the arrays and calculated at selected positions as a function of the array periodicity and ellipse thickness. Note, that at the onset of switching the interaction between the ellipses serves to reinforce the outside magnetic field used for switching the magnetization.

The size of the array also has an effect on the interaction strength. When the size of a square array, with lattice constant $c$, increases from $N \times N$ to $(N+1) \times(N+1)$ the number of ellipses increases by $2 N+1$ at a distance of $r \approx \frac{(2 N+1) c}{2}$ from the center of the array. The near-field term decays as $r^{-3}$. Thus, the incremental contribution to the interaction field 


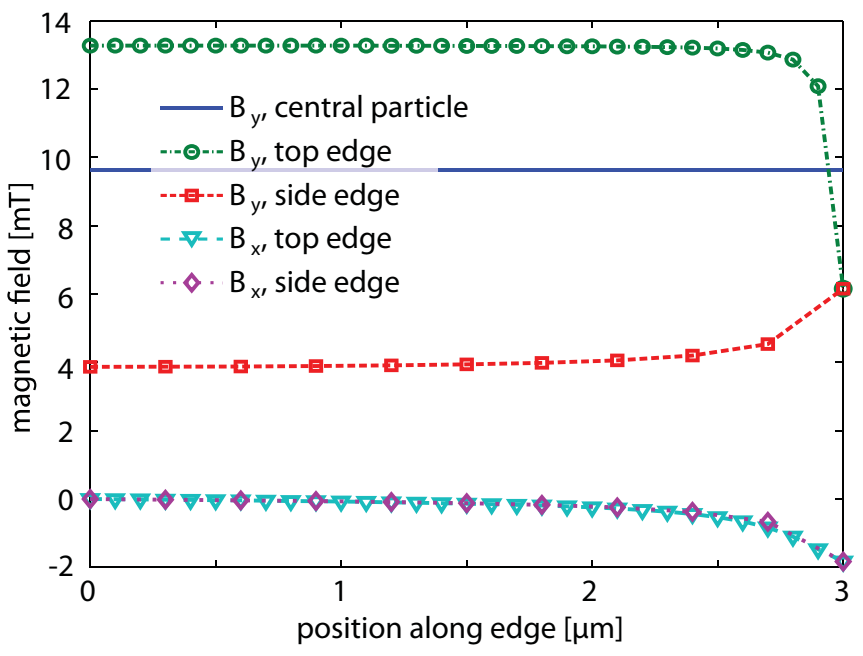

FIG. 11. (Color online) Magnetic field at the geometrical centers of $150 \mathrm{~nm} \times 50 \mathrm{~nm}$ ellipses in an array with a short axis period $100 \mathrm{~nm}$ in the $x$ direction and thickness of $10 \mathrm{~nm}$; there are $61 \times 21$ particles in the array. The fields are plotted along the edges of the array from their respective centers $(0 \mu \mathrm{m})$ to the corner $(3 \mu \mathrm{m})$ with markers indicating positions of the particles along the edges (there are more particles along the top edge, hence the density of markers is larger than at the side edge). The solid line marks the magnetic field (only $B_{y}$ component is nonvanishing) acting onto the central particle. At the edges the $B_{y}$ field is either smaller (side edge) or larger (top edge, except corner) than at the center of the array. There is also a nonvanishing $B_{x}$ component which is strongest at the corner and decays rapidly when moving away from it. decreases as $N^{-2}$ or $r^{-2}$, and the field approaches a limiting value. For example, for a 10-nm-thick ellipse the magnetic field acting onto the central particle in a square array $600 \mathrm{~nm} \times$ $600 \mathrm{~nm}(3 \times 7$ ellipses $)$ is approximately $11 \mathrm{mT}$, a value which decreases when increasing the array size. In the limit of large arrays it is equal to approximately $9.3 \mathrm{mT}$. This value is reached (within a $1 \%$ accuracy) already for arrays $6 \mu \mathrm{m} \times$ $6 \mu \mathrm{m}$ (the employed $61 \times 21$ particle arrays). The magnitude of this effect as well as the trend (increase or decrease) depend on the array geometry. The above-mentioned effect (decrease of the interaction field with array size) occurs for a periodicity aspect ratio that is the same as that of the ellipse's semiaxes, namely 3 . However, for the same ellipses arranged in a square lattice with a period of, e.g., $150 \mathrm{~nm}$, increasing the array size will asymptotically increase the interaction field. Moreover, the periodicity will also affect the sense of the magnetic field generated by the array. For the previously mentioned square lattice the interaction field will change qualitatively-it will reinforce the magnetization and, as mentioned, will increase in amplitude for larger arrays. Figure 13 shows how the interaction field varies with the aspect ratio for two cases of array configurations. Thus, by designing the array properly, it is possible to tailor the magnetization properties to either promote switching (by lowering the switching field via the interaction field) or inhibit it.

\section{E. Micromagnetic simulation of arrays}

To find the influence of interactions on the magnetization switching in an array it is not sufficient to simply add an
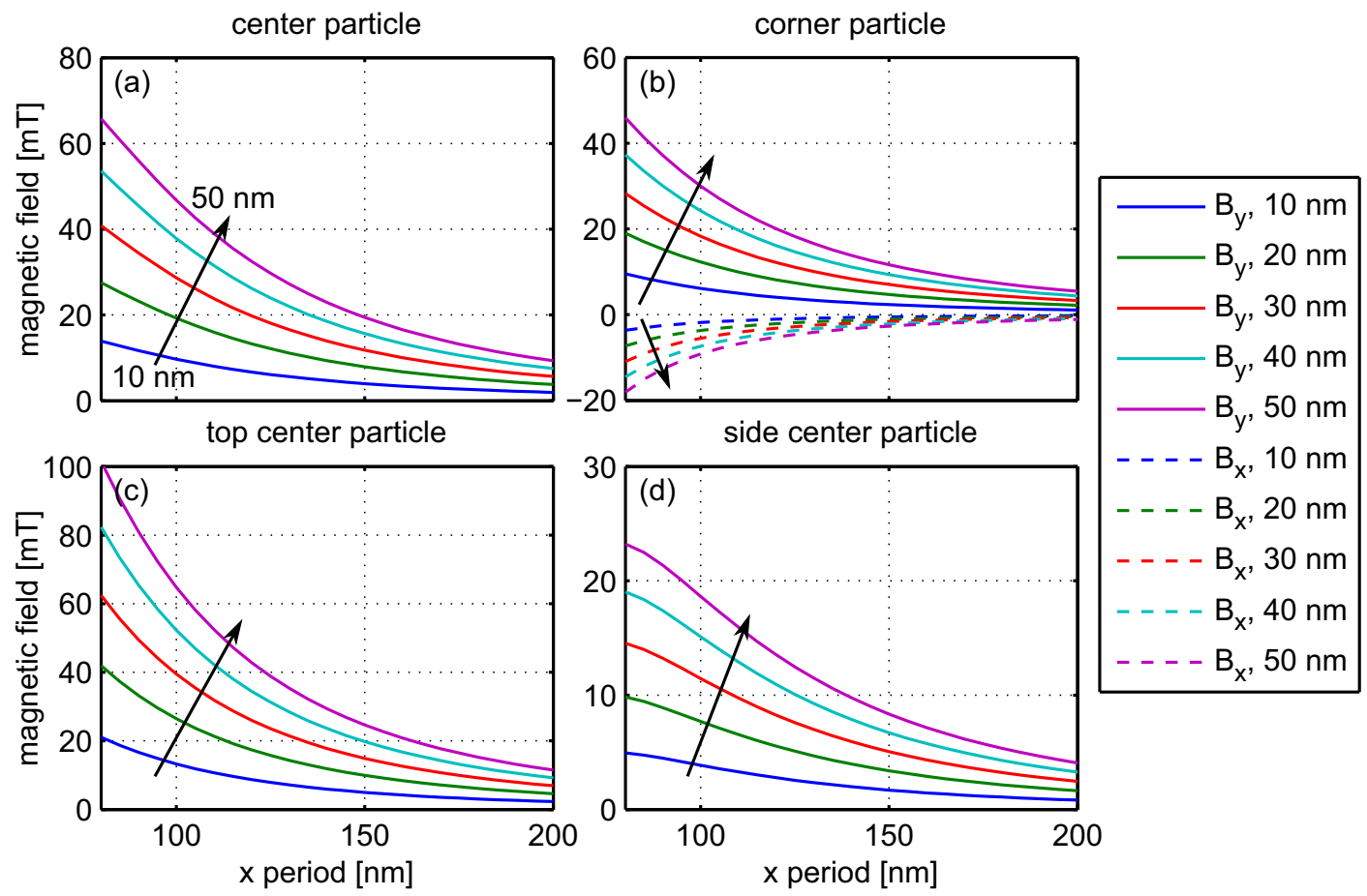

FIG. 12. (Color online) Dependence of the magnetic field acting onto the nanoellipses on the array periodicity and ellipse thickness. The field is calculated for (a) the central particle, (b) the corner particle, (c) the top central particle, and (d) the side central particle. In all cases the solid lines indicate the $B_{y}$ component which is parallel to the magnetization of the ellipses and for the corner particle the dashed lines show the $B_{x}$ component. The arrows indicate the direction of increasing thicknesses from 10 to $50 \mathrm{~nm}$. 


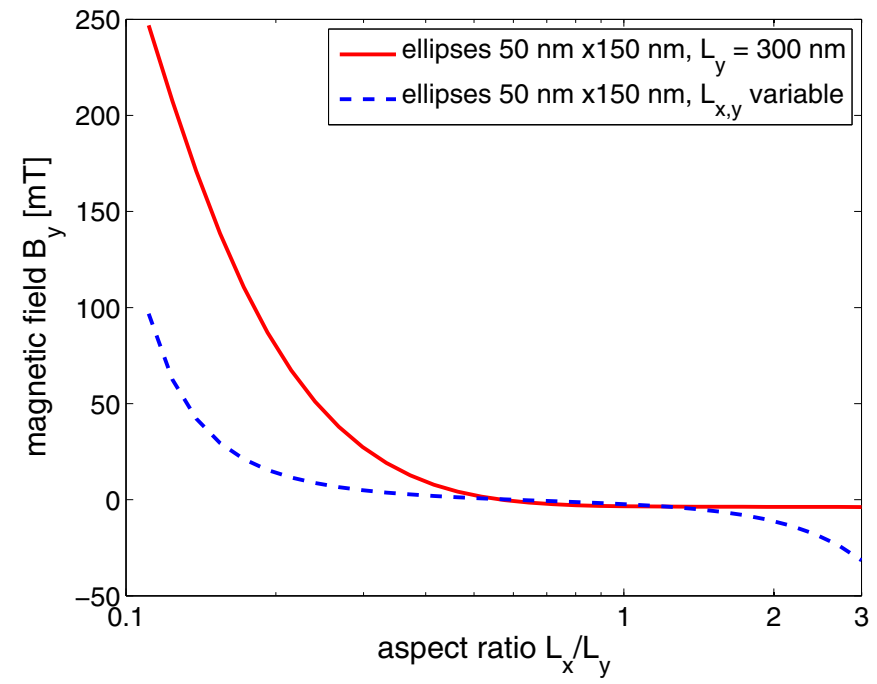

FIG. 13. (Color online) The interaction field calculated at the center of an array, for two cases of varying aspect ratios. The arrays comprise $11 \times 11$ nanoparticles, all $20 \mathrm{~nm}$ thick and with lateral sizes as noted in the figure. $L_{x}$ and $L_{y}$ are the periods in the $x$ and $y$ directions, respectively. The aspect ratio $A=L_{x} / L_{y}$ is varied according to the following relations. For one set of ellipses $L_{y}$ is const $=300 \mathrm{~nm}$ and $L_{x}=L_{y} A$. In the second case, for the same size of ellipses, $60 \mathrm{~nm} \leqslant L_{x} \leqslant 480 \mathrm{~nm}$, and $L_{y} / A$. The magnetic moment of each nanoparticle is assumed to be in the $-y$ direction.

effective field to the applied field, since this does not take the local variation of the interaction into account, nor the changes of the magnetic domain structure of the ellipses during demagnetization. One method, often applied when looking for ferromagnetic resonances (FMR) in arrays, is to find the effects of dipolar interactions by simulating the array as a single ellipse under periodic boundary (PB) conditions. Figure 14(a) shows the resulting micromagnetic state at the last field step before switching for the smallest, 10-nm-thick ellipses. (The image displays one of the three identical layers in the simulation.) With open boundary (OB) conditions switching occurred at $B_{\mathrm{sw}}=354 \mathrm{mT}$. Simulation with PB for wide and narrow separation yielded switching at 345 and $331 \mathrm{mT}$, respectively. This gives an estimate of interaction field strengths of 9 and $23 \mathrm{mT}$, respectively, in agreement with our calculated interaction fields. An additional, important effect of interactions can be noted in Fig. 14(a); the symmetry of the magnetic state just before switching is changed from being an $S$ state to more like a $C$ state.

A more realistic picture is expected from simulations of the actual array. We did not make a complete investigation to catch the statistics and all details of how array size and step length during demagnetizing influence the processes; here we give a few examples. The smallest, 10-nm-thick ellipses were studied in, e.g., small arrays with $3 \times 3,5 \times 5,4 \times 6$, and $6 \times$ 4 ellipses in configurations with narrow and wide separation. In all cases the first reversal occurs in fields lower than $B_{\mathrm{sw}}$ for the corresponding single ellipse. In arrays with wide separation the ellipses switch individually and the switching seems to always be finalized at about $B_{\mathrm{sw}}$. With narrow separation the switching range is narrower.

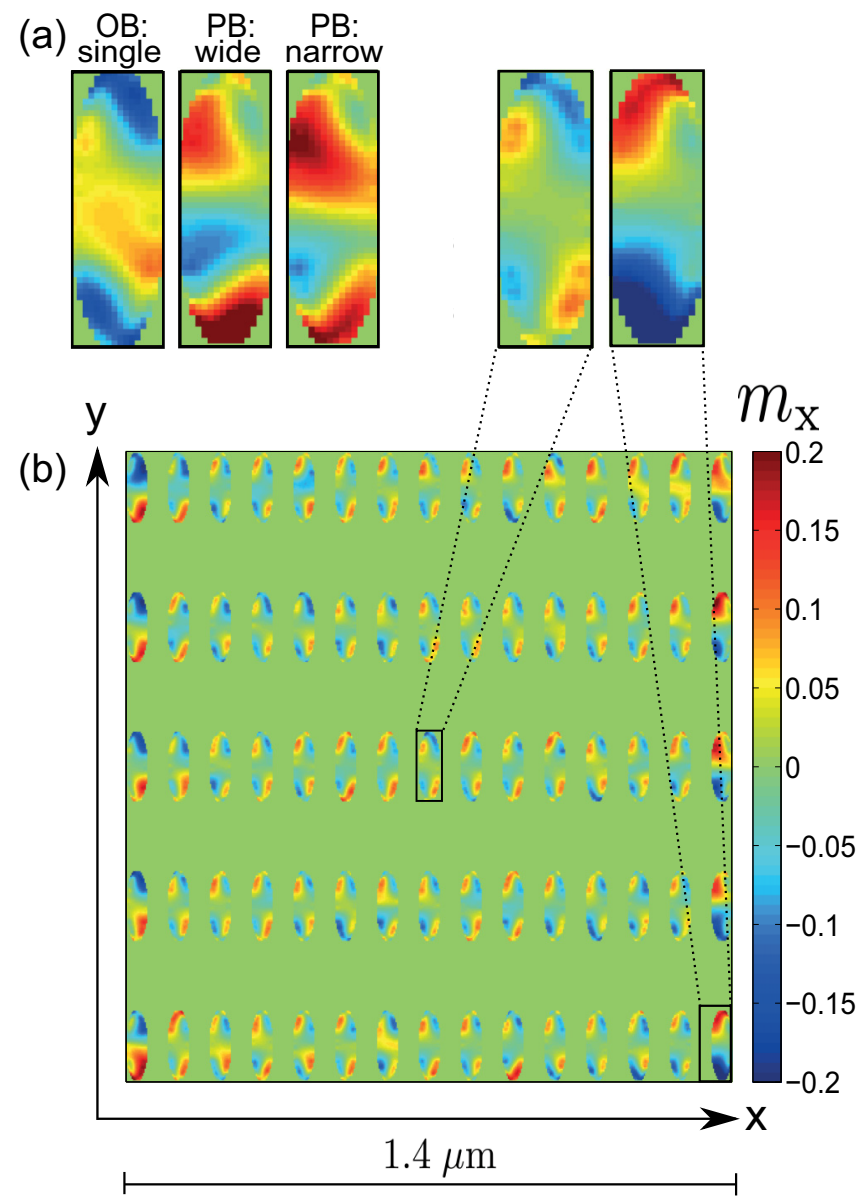

FIG. 14. (Color online) (a) Comparison of simulated states of a $48 \mathrm{~nm} \times 144 \mathrm{~nm}$ single ellipse with $t=3 \times 3 \mathrm{~nm}$, just before switching. From left to right, the $x$ component of the magnetization was obtained with open boundary conditions and periodic boundary conditions for wide and narrow separation, respectively. (b) The $x$ component of the magnetization in the simulated state of an array with $15 \times 5$ of the same ellipses. Layer 1 of three is shown in all figures. The field is at the point before the switching of the first ellipse, located in the lower right corner of the array.

Figure 14(b) shows one step at $B=309.1 \mathrm{mT}$ during demagnetization of a larger array of $15 \times 5$ ellipses with narrow separation. In the next step, with $B=311.6 \mathrm{mT}$, the first ellipse at the lower right corner is reversed and in the following $B=314.1 \mathrm{mT}$ all ellipses are reversed. The variations in internal structure, with strong effects at the edges of the array, can be noted. A simulation of a still larger array of the same configuration, $54 \times 18$ ellipses in field steps of $1.25 \mathrm{mT}$, displayed a switching of all ellipses at $B=310.4 \mathrm{mT}$. The switching fields of these two arrays are both about $40 \mathrm{mT}$ lower than $B_{\mathrm{sw}}$ of the single Fe ellipse. The difference is larger than the calculated interactions fields, which implies that effects of variations in local interaction fields and internal domain structures are important. Although we did not perform simulations for the larger ellipses, it is interesting to note that the difference between $B_{\mathrm{sw}}$ and $B_{\mathrm{cl}}$ is of the order 50-60 mT for these, as discussed in Sec. III A. The simulations of the arrays support the conclusion drawn from the calculation of 
the interaction fields, namely that the interactions saturate for arrays with sizes in these ranges.

\section{DISCUSSION}

The properties of a $2 \mathrm{D}$ array of submicron or nanosize ferromagnetic particles are determined by the combination of, on one hand, the domain state and magnetization reversal processes of each single particle, and on the other, the array configuration. Now, the question is how well theoretical models can describe the magnetic properties of the real system, or perhaps whether an ideal system really can be fabricated.

\section{A. Magnetization reversal}

The magnetization switching field $B_{\mathrm{cl}}$ was determined for arrays of epitaxial $\mathrm{Fe}(001)$ ellipses with thickness $t$ in the range $10-50 \mathrm{~nm}$ and lateral sizes in the range $50-450 \mathrm{~nm}$. It was found that $B_{\mathrm{cl}}$ decreases with increasing particle size, and the $t$ dependence of $B_{\mathrm{cl}}$ for each particle size displays a maximum in the range $20-25 \mathrm{~nm}$. This thickness is of the order of the wall width parameter $l_{\mathrm{w}}=22 \mathrm{~nm}$. It is not possible to fully characterize the magnetization reversal from the major hysteresis loops only. The switching field range and remanent domain state were determined also from MFM observation of dc demagnetization. The equilibrium zero-field state during these processes was observed to be SD, except for the largest particles, which were occasionally seen as bidomains. Complementary measurements of the hysteresis with FORC analysis could clearly identify the field range for single-particle reversal and sort out effects of interactions. It can be noted that in the literature there are several examples of hysteresis curves that have similar character, but are generated by different materials and magnetization processes. Pot bellies, with spreading middles, and wasp waists, with constrained middles, were shown by Tauxe et al. [24] to originate from different populations of superparamagnetic and single-domain particles. Bennett and Della Torre [28] showed how two exchange coupled materials may be arranged to yield one or the other of the two shapes, and how FORC analysis could separate the two cases.

The switching field $B_{\mathrm{sw}}$ of single Fe ellipses was determined by micromagnetic simulations, in which the values for bulk Fe were used as input parameters. The size and thickness dependence of $B_{\mathrm{cl}}$ observed in the experiments is well reproduced in the behavior of the single ellipses. The absolute values display systematic deviations, with $B_{\mathrm{cl}}$ being lower than corresponding $B_{\mathrm{sw}}$. For the $100 \mathrm{~nm} \times 300 \mathrm{~nm}$ ellipses with $400 \mathrm{~nm} \times 1200 \mathrm{~nm}$ separation $B_{\mathrm{cl}}$ is in the range 100-200 mT, about $50 \mathrm{mT}$ lower than $B_{\mathrm{sw}}$ for corresponding thicknesses. For the smaller and larger ellipses the difference is smaller and larger, respectively. For the single ellipses it was shown that below the maximum of $B_{\mathrm{sw}}$ at $t_{\mathrm{sw}}$, about equal to $l_{\mathrm{w}}$, all layers undergo identical in-plane reversals, and the micromagnetic state of a single ellipse immediately before the final switching of the dominant magnetization direction is an $S$-like quasisingle domain state. When interactions are taken into account by introducing periodic boundary conditions, the symmetric $S$ state is deformed towards a more $C$-like state. In simulated arrays the particles display a variety of similar, but not identical, $C$ - or $S$-like states. The FORC analysis gave evidence that, whereas details in the major loops resembled those of nanostructures reversing via vortex states, the FORC distributions showed no signs of vortex reversal. Altogether, our interpretation is that the particles undergo in-plane switching through the development of the quasisingle $C$ or $S$ states in which the spins finally rotate into the reversed SD state. Above $t_{\mathrm{sw}}$ the switching becomes more complex with a gradually increasing phase shift between the in-plane rotation in different layers, and finally also an out-of-plane component develops.

These switching processes are known to be sensitive to imperfections in the materials, as well as to interactions between the particles. The influence of interactions are discussed in the next section. The imperfections in the materials may be defects and variations - on one hand in the structure and/or surfaces of the Fe films on the other in the particle shapes and edges. Particle edge effects of the size of a discretization cell $(3 \mathrm{~nm})$ were found to decrease the switching by the order of $10 \mathrm{mT}$. We did not study the influence of capping layer directly, but note that in our earlier studies of larger Fe/Co particles capped with $\mathrm{V}$, the relative saturation remanence was higher [23]. Fruchart et al. [29] showed hysteresis curves with higher squareness in their studies of $\mathrm{Fe}(110)$ dots. Their films were sandwiched between two Mo or W (110) layers. Our capping layers of $\mathrm{Al}_{2} \mathrm{O}_{3}$ were chosen because they prevent oxidation efficiently; examples showed identical hysteresis curves when the measurements were repeated after three years.

\section{B. Arrays}

There are two important conclusions to draw from the calculations of the interaction field in the arrays. The character of the interaction, whether it promotes or delays magnetization reversal, is determined by the aspect ratio of the array grid, and the interaction strength saturates as the size of the array increases. The dipolar interaction field was calculated for arrays with $61 \times 21$ Fe ellipses with lateral size $50 \mathrm{~nm} \times$ $150 \mathrm{~nm}$ and thickness in the range $10-50 \mathrm{~nm}$. For these the thickness dependence of the field was calculated at different positions, in the center and at the edges, and for varying periodicity while keeping the aspect ratio $1: 3$ of the grid. In this particular grid the interactions promote the onset of switching, as was clearly demonstrated in both experiments and micromagnetic simulations; the switching field of arrays was in all cases lower than that of the corresponding single ellipses. For the smallest particles the interaction field is of the order of $10 \mathrm{mT}$. For 50-nm-thick $100 \mathrm{~nm} \times 300 \mathrm{~nm}$ ellipses with $200 \mathrm{~nm} \times 600 \mathrm{~nm}$ separation, the calculated interaction field $22.6 \mathrm{mT}$ at the center of the array agrees well with the average interaction field $\approx(18 \pm 2) \mathrm{mT}$, determined by FORC analysis.

Micromagnetic simulations were made for arrays of $15 \times 5$ and $54 \times 18$ ellipses with lateral size $50 \mathrm{~nm} \times 150 \mathrm{~nm}$ and $10 \mathrm{~nm}$ thickness. The switching fields are about the same for the two arrays, thus showing the same tendency of saturation as found for the calculated interaction field. We compared the switching fields obtained by simulations with periodic boundary conditions and arrays with the value $B_{\text {sw }}$ obtained for corresponding single ellipse. Whereas the 
shift obtained with periodic boundary conditions is in good agreement with the calculated interaction field, the shift for the arrays is significantly larger. We suggest this to be an effect of interactions on the magnetic domain states during switching of the ellipses. It is appropriate to here point out the limitations of the quasistatic treatment, since it cannot give full account of the reversal processes. Thermal fluctuations and statistical processes in the magnetization reversal are always present, but were not considered in our calculations. In another work [30] the magnetization dynamics of the samples was also investigated. In ferromagnetic resonance (FMR) the behavior is dominated by the properties of the single Fe ellipses, without significant influence of interactions.

\section{CONCLUSIONS}

The experimental and numerical results yield a consistent picture of the studied arrays of Fe ellipses: the magnetization reversal, occurring in the range $100-400 \mathrm{mT}$, is mainly determined by the properties of the corresponding single Fe ellipses, and the interaction fields determined by the array configuration are of the order of tens of mT. For the actual arrays the interactions promote switching. For film thicknesses below the wall width parameter of $\mathrm{Fe}$, about $20 \mathrm{~nm}$, magnetization reversal occurs without formation of domain walls or vortices. In this range an array might be tuned to obtain a well-defined switching field. Another parameter to consider is the size of the array. The finite arrays with $54 \times 18$ and $61 \times 21$ ellipses are only $5 \mu \mathrm{m} \times 5 \mu \mathrm{m}$ and $6 \mu \mathrm{m} \times 6 \mu \mathrm{m}$ large. The interaction effects are already stabilized and the majority of ellipses feel the same interaction field as the central particle. This implies that it could be possible to arrange several substructures of arrays with different well-defined switching fields, within limited areas on one chip.

\section{ACKNOWLEDGMENT}

M.H. is grateful for the support of Prof. Peter Apell.
[1] See, e.g., the review by B. D. Terris and T. Thomson, Nanofabricated and self-assembled magnetic structures as data storage media, J. Phys. D 38, R199 (2005).

[2] V. V. Kruglyak, S. O. Demokritov, and D. Grundler, Magnonics, J. Phys. D 43, 264001 (2004).

[3] Magnonics, Topics in Applied Physics No. 125, edited by S. O. Demokritov and A. N. Slavin (Springer-Verlag, Berlin, Heidelberg, 2013).

[4] V. Kapaklis, U. B. Arnalds, A. Farhan, R. V. Chopdekar, A. Balan, A. Scholl, L. J. Heyderman, and B. Hjörvarsson, Thermal fluctuations in artificial spin ice, Nat. Nanotechnol. 9, 514 (2014).

[5] R. L. Stamps and R. E. Camley, Magnetization processes and reorientation transition for small magnetic dots, Phys. Rev. B 60, 11694 (1999).

[6] M. A. Kayali and W. M. Saslow, Hysteresis of finite arrays of magnetic nanodots, Phys. Rev. B 70, 174404 (2004).

[7] Y. Takagaki and K. H. Ploog, Magnetization of two-dimensional square arrays of nanomagnets, Phys. Rev. B 71, 184439 (2005).

[8] M. Alcántara Ortigoza, R. A. Klemm, and T. S. Rahman, Comment on "Magnetization of two-dimensional square arrays of nanomagnets", Phys. Rev. B 74, 226401 (2006).

[9] M. Ewerlin, D. Demirbas, F. Brüssing, O. Petracic, A. A. Ünal, S. Valencia, F. Kronast, and H. Zabel, Magnetic Dipole and Higher Pole Interaction on a Square Lattice, Phys. Rev. Lett. 110, 177209 (2013).

[10] R. Luttge, Massively parallel fabrication of repetitive nanostructures: Nanolithography for nanoarrays, J. Phys. D 42, 123001 (2009).

[11] See, e.g., the review by K. Yu. Guslienko, Magnetic vortex state stability, reversal and dynamics in restricted geometries, J. Nanosci. Nanotechnol. 8, 2745 (2008).

[12] V. Novosad, K. Yu. Guslienko, H. Shima, Y. Otani, S. G. Kim, K. Fukamichi, N. Kikuchi, O. Kitakami, and Y. Shimada, Effect of interdot magnetostatic interaction on magnetization reversal in circular dot arrays, Phys. Rev. B 65, 060402(R) (2002).

[13] Y. Wang, W. H. Shi, H. X. Wei, D. Atkinson, B. S. Zhang, and X. F. Han, Manipulation of magnetization reversal of $\mathrm{Ni}_{81} \mathrm{Fe}_{19}$ nanoellipse arrays by tuning the shape anisotropy and the magnetostatic interactions, J. Appl. Phys. 111, 07B909 (2012).

[14] M. Pardavi-Horvath, Interaction effects in magnetic nanostructures, Phys. Status Solidi A 211, 1030 (2014).

[15] M. Hanson, C. Johansson, B. Nilsson, P. Isberg, and R. Wäppling, Magnetic properties of two-dimensional arrays of epitaxial Fe(001) submicron particles, J. Appl. Phys. 85, 2793 (1999).

[16] M. Hanson, O. Kazakova, P. Blomqvist, R. Wäppling, and B. Nilsson, Magnetic domain structures in submicron-size particles of epitaxial $\mathrm{Fe}(001)$ films: Shape anisotropy and thickness dependence, Phys. Rev. B 66, 144419 (2002).

[17] M. Hanson, R. Bručas, and O. Kazakova, Effects of size and interactions on the magnetic behaviour of elliptical (001)Fe nanoparticles, J. Magn. Magn. Mater. 316, 181 (2007).

[18] MicroMagus, supplied by MATESY, Magnetic Technologies and Systems GmbH.

[19] A. Hubert and R. Schäfer, Magnetic Domains: The Analysis of Magnetic Microstructures (Springer-Verlag, Berlin, Heidelberg, 1998).

[20] W. Rave and A. Hubert, Magnetic ground state of a thin-film element, IEEE Trans. Magn. 36, 3886 (2000), and references therein.

[21] J. E. Miltat and M. J. Donahue, Numerical micromagnetics: Finite difference methods, in Micromagnetism, Handbook of Magnetism and Advanced Magnetic Materials Vol. 2, edited by H. Kronmüller and S. Parkin (Wiley-Interscience, Chichester, 2007), pp. 742-764.

[22] J. M. D. Coey, Magnetism and Magnetic Materials (Cambridge University Press, Cambridge, 2010). 
[23] O. Kazakova, M. Hanson, P. Blomqvist, and R. Wäppling, Interplay between shape and magnetocrystalline anisotropies in patterned bcc Fe/Co(001) multilayers, Phys. Rev. B 69, 094408 (2004).

[24] L. Tauxe, T. A. T. Mullender, and T. Pick, Potbellies, wasp-waists, and superparamagnetism in magnetic hysteresis, J. Geophys. Res. 101, 571 (1996).

[25] C. R. Pike, A. P. Roberts, and K. L. Verosub, Characterizing interactions in fine magnetic particle system using first order reversal curves, J. Appl. Phys. 85, 6660 (1999).

[26] R. K. Dumas, C.-P. Li, I. V. Roshchin, I. K. Schuller, and Kai Liu, Magnetic fingerprints of sub-100 nm Fe dots, Phys. Rev. B 75, 134405 (2007).
[27] D. A. Gilbert, G. T. Zimanyi, R. K. Dumas, M. Winklhofer, A. Gomez, N. Eibagi, J. L. Vicent, and K. Liu, Quantitative decoding of interactions in tunable nanomagnet arrays using first order reversal curves, Sci. Rep. 4, 4204 (2014).

[28] L. H. Bennett and E. Della Torre, Analysis of wasp-waist hysteresis loops, J. Appl. Phys. 97, 10E502 (2005).

[29] O. Fruchart, J.-P. Nozières, W. Wernsdorfer, D. Givord, F. Rousseaux, and D. Decanini, Enhanced Coercivity in Submicrometer-Sized Ultrathin Epitaxial Dots with In-Plane Magnetization, Phys. Rev. Lett. 82, 1305 (1999).

[30] V. Flovik, F. Macià, J. M. Hernàndez, R. Bručas, M. Hanson, and E. Wahlström, Tailoring the magnetodynamic properties of nanomagnets using magnetocrystalline and shape anisotropies, Phys. Rev. B 92, 104406 (2015). 